The articles are handsomely printed on glossy paper, so that micrographs are excellently reproduced. Their content is typically a mixture of technique and application. About a quarter of each issue is taken up with book reviews, news and commentary, with advance information on a variety of conferences. All aspects of microscopy and microanalysis are covered, although electron microscopy dominates at the moment. There is a lively correspondence section.

Any institution outside America with research programmes in microscopy should have access to a copy of this journal. Active members of the North American community will want to become regular readers.

L. M. Brown is at the Cavendish Laboratory, University of Cambridge, Madingley Road, Cambridge CB3 OHE, UK.

\section{Digging up the recent past}

\section{International Journal of Historical Archaeology}

Editor Charles E. Orser Jr.

Plenum Press. 4/yr. USA \$100, elsewhere

$\$ 115$ (institutional); \$35 (personal)

\section{Frans Verhaeghe}

The archaeologies of the medieval, industrial and particularly early modern periods are relative newcomers, the latter being the most recent addition. Since the 1960s, it has been steadily growing, notably in the AngloSaxon world. Elsewhere in Europe, the importance and potential of sixteenth- to eighteenth-century remains are now also slowly being recognized. Journals such as Post-Medieval Archaeology and Historical

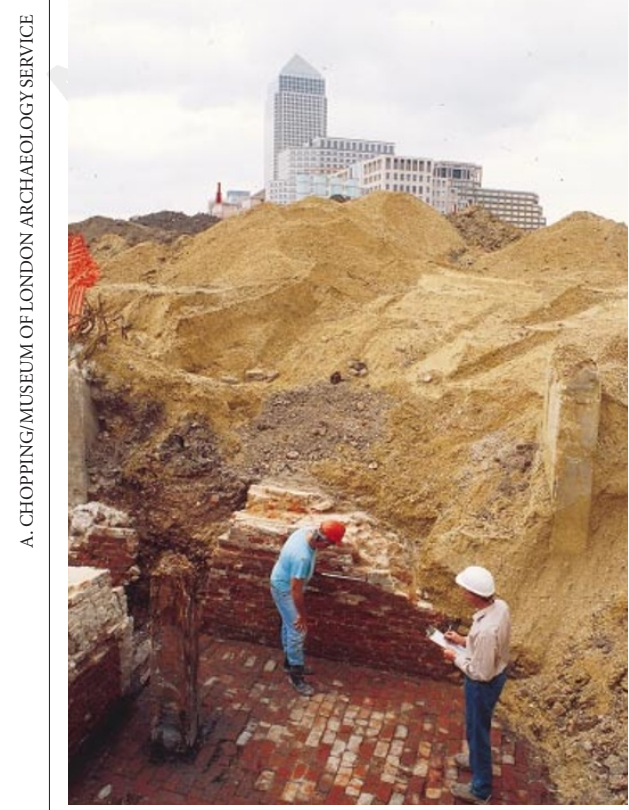

A dig at Lime Kiln Dock in London, overlooked by the tower of Canary Wharf.
Archaeology (published since 1967 by the UK Society for Post-Medieval Archaeology and the US Society for Historical Archaeology respectively) have created models, but there is room for more international approaches as well as more theoretical and applied research. Hence this new journal.

Judging from the editor's introduction, the journal's target area is the archaeology of any 'historical' period in any region of the planet. It offers medium-sized to more extensive papers, from about 10 to 45 pages long. Some are reprints of less readily accessible papers, but most of the 20 -odd papers now published are new, and all bar one focus on the past half-millennium and on the formerly colonized world outside Europe. The contributions are well presented and seem to be published fairly rapidly, in a handy $23 \mathrm{~cm}$ by $15 \mathrm{~cm}$ standard format, on paper allowing for good drawings and reasonable (though not high-quality) photographs.

A 'Views and Commentaries' section presents innovative and thought-provoking statements on issues confronting archaeologists of historical periods. Reviews and book reviews are not (yet?) offered but comments on published papers occasionally are.

The contents and scope of the first two volumes are very satisfying to anyone interested in ethnic and social issues in archaeology, including the impact of colonization and the role played by different components of material culture in shaping early modern history in many parts of the world.

Apart from the very useful information, I find the most stimulating feature to be the strong direct or indirect emphasis on methodology and theory in archaeology and material culture studies. This contributes not only to a better understanding of our recent past but also to the development of archaeology as a whole. This is why the series gives real value at a very reasonable price. $\square$ Frans Verhaeghe is in the Department of Art History and Archaeology at the Free University of Brussels, Pleinlaan 2, B-1050 Brussels, Belgium.

\section{Free advice for experimenters}

\begin{tabular}{l}
\hline Technical Tips Online \\
Managing editor Mark Patterson \\
Elsevier. On-line only, free. \\
http://tto.trends.com \\
Laurie Goodman
\end{tabular}

Web-surfing scientists have a new reason to catch electronic waves. The fully - and solely - on-line journal Technical Tips Online provides quick, easy access to optimal methods for doing what researchers do best: experiments. Two great things about the site: it's free, and it's peer-reviewed. It therefore satisfies every web-surfer's belief that everything on the Internet should be free, and meets the need for high scientific standards for online-only resources.

Technical Tips Online is a fusion of a peerreviewed techniques journal and a methods database. It contains three types of article: peer-reviewed 'Technical Tips' (novel methods or major advances on known methods); non-peer-reviewed 'Protocols' (editorially invited protocols from laboratories with expertise in the method); and non-peerreviewed 'Application Notes' (companysubmitted paid-for reports detailing product use). Editorially selected comments from readers, which are quite fun to read, some with author responses, are also available.

The site is updated fortnightly and is extremely user-friendly. One can browse by category - Polymerase Chain Reaction, Electrophoresis, Microbiology, Purification Methods, Gene Expression, Microscopy, Tissue Culture, and Cloning and Sequencing - or examine only the latest additions. Using its comprehensive search engine is definitely superior to scanning several articles or journals for basic protocols, and then trying to glean modifications in later publications. After a while, however, this site could become equally unwieldy, because it might have to be searched just as extensively to obtain all useful protocol modifications. Given the ease of changing on-line text (which is what makes on-line protocols so appealing), this shouldn't be allowed to occur. Periodic processing of related information by the editors into a single update would increase this site's long-term value.

In this regard, it will be interesting to see how the site develops. Sustaining and properly maintaining any dedicated database is an enormous task, requiring time and money (it is supported by advertisements). All in all, however, if the site is well-maintained and the number of core protocols and useful modifications increases at a rapid rate, this site is well worth a bookmark in my book - er, computer.

Laurie Goodman is at Cold Spring Harbor Laboratory, 1 Bungtown Road, Cold Spring Harbor, New York 11724, USA. \section{Fuel for thought}

\section{Combustion Theory and Modelling}

Editors-in-chief Bill Dold and Mitch

Smooke

Institute of Physics. 4/yr. USA \$257, elsewhere $£ 127$ (institutional); USA \$97, elsewhere $\mathfrak{E} 48$ (personal)

\section{A.C.Mclntosh}

Interest in combustion research has grown in recent years because of public pressure to find cleaner, more efficient means of energy production, which will all involve combustion of some kind for generations to come. 
Because of the nonlinear features of the chemical reactions involved, a whole branch of mathematics has arisen to model the complex behaviour of solids, liquids and gases which are undergoing combustion. This, coupled with great advances in numerical approaches, has led to the formation of a new journal specifically devoted to the "application of mathematical theory, modelling, numerical simulation and experimental techniques to the study of combustion". The inclusion of experimental techniques in its scope is laudable and, it is hoped, will encourage greater involvement of experimentalists in the journal.

Combustion Theory and Modelling has been published since March 1997 and has received papers on a wide area of topics, such as flame stability, turbulent combustion, thermal explosion, compressible reacting flows, detonations and filtration combustion. In that a growing number of authors are making use of the journal, it is evident that it is taking a leading place in the status of archived combustion literature. Furthermore, it is readily available at competitive prices for personal subscription and, most importantly, for library subscription. It is the relative cheapness of library availability that should be most attractive in the present financial climate of universities in the UK and world-wide.

The high profile of theory and modelling in this quarterly is particularly welcome. The study of combustion fundamentals is vital to making progress in our understanding of practical combustion systems. Too often the tendency in research has been to regard theory as either too difficult, or naively to regard computation as somehow having replaced the need for modelling altogether. Advance in any scientific research area is connected to the progress of experiment (numerical or laboratory) coupled with testable theory. That way physical principles are uncovered and light the way for further advance.

A. C. McIntosh is in the Department of Fuel and Energy, University of Leeds, Leeds LS2 9JT, UK.

\section{Golden age journal}

Multibody System Dynamics

Editor Werner Schiehlen

Kluwer Academic Publishers. 4/yr. \$241.50

(institutional), $\$ 85$ (personal)

\section{John Hogan}

Contemporary mechanics traces its origins to Newton's laws of motion. Traditionally divided into fluid and solid mechanics, the theoretical side in the early part of this century saw ideas such as boundary layer theory give analytic tractability to the otherwise impenetrable continuum equations in these areas. Enormous strides in computer power mean that problems such as the large-scale computation of global weather patterns can now be addressed. More recently, the qualitative approach crucial to understanding nonlinear dynamics (including 'chaos theory') has been embraced by mechanics to the extent that the subject may be on the verge of a golden age, in which fields previously thought impenetrable can be explored.

The international body in the subject is the International Union of Theoretical and Applied Mechanics (IUTAM). Its president, Werner Schiehlen, is the editor of Multibody System Dynamics, which is devoted to one of the most important new fields. Examples of such systems include nearly every man-made object of engineering importance, such as cars, lorries, railway vehicles and robots.

Looking through the articles in this journal, it is clear that the equations of motion can be written down, that computers can produce solutions and that, given enough parameters, sufficient agreement can be found with observation. But something is missing. There seems to be no visceral, intuitive understanding of the behaviour of such systems - with their enormous numbers of degrees of freedom - especially when subject to constraints. Of course this is a highly non-trivial problem, but without a 'feel' for a problem, progress is liable to be restricted. The success and even survival of the journal may well be directly related to this issue.

Nevertheless, Schiehlen is to be congratulated for highlighting this area, and Kluwer applauded for its faith in him. Readers of Nature interested in this field should start with Schiehlen's comprehensive review in Multibody System Dynamics 1, 149-188 (1997), and then attend IUTAM's congress in Chicago in August 2000.

John Hogan is in the Department of Engineering Mathematics, Queen's Building, University Walk, Bristol BS8 1TR, UK.

\section{Living on the edge}

\section{Extremophiles: Life Under Extreme Conditions}

Editors Koki Horikoshi, Garo Antranikian, William D. Grant, Terry A. Krulwich and Juergen Wiegel

Springer. 4/yr. \$267, ¥25,000, DM380

\section{John A. Baross}

Why do we suddenly need a journal dedicated to micro-organisms that grow under conditions we humans define as extreme? Micro-organisms that grow in saturated concentrations of salt have been studied for almost 80 years. Pressure- and cold-loving bacteria were identified from the deepest ocean trenches during the Danish Galathea expedition in 1952. For at least 20 years we have known of micro-organisms that grow at temperatures close to $100{ }^{\circ} \mathrm{C}$, alkalinities exceeding $\mathrm{pH} 10$, and acidities near $\mathrm{pH} 0$.

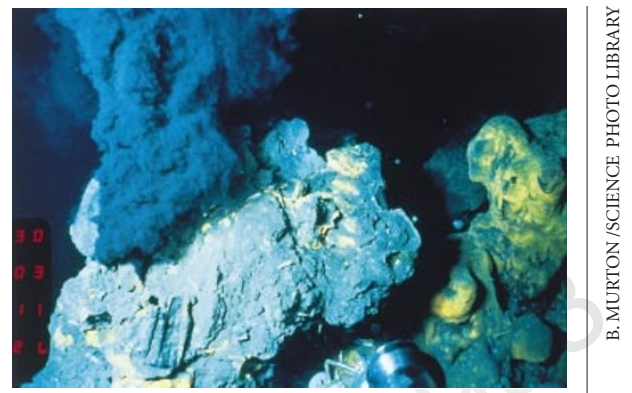

The Saracen's Head 'black smoker' vent in the Atlantic Ocean floor.

Yet over the past ten years extremophiles have taken on a life of their own, no longer studied as academic peculiarities. High- and low-temperature enzymes from thermal extremophiles are exploited for their many biotechnological applications, while acidophiles assist in the mining of precious metals from complex minerals. Global phylogenetic trees based on nucleic acid sequences indicate that hyperthermophiles are the oldest of extant organisms, holding potential clues to the origin of life on Earth. Their physiologies suggest that they could grow on other solar bodies or survive transit between them. Such controversial ideas, spurred most recently by evidence that the Jovian moon, Europa, may have a liquid-water ocean, are very much part of the study of extremophiles and the emergent field of 'astrobiology'. Life in extreme environments is in vogue.

Against this backdrop, the creation of a journal dedicated to extremophiles was inevitable. Its appearance is also an acknowledgement of the growing community of researchers dedicated to studying these very exciting organisms from a wide range of disciplinary perspectives. This growth is reflected in the diversity of research papers in the first four issues reporting novel and important new discoveries. The topics range from descriptions of new organisms and physiologies to the characterization of their enzymes and molecular biology. One can also learn that the highest temperature for growth by a pure culture has risen from 110 to $113^{\circ} \mathrm{C}$, that there exist organisms that can oxidize hydrogen gas using ferric-iron as the electron acceptor, and that physiologically diverse heterotrophs exist in the deepest trenches of the ocean.

Overall, the research articles in the first four issues are of high quality. Three issues also offered a very useful review article: I recommend that the editors continue to solicit reviews that keep the community informed of current trends in this rapidly changing field. This attractively produced journal is essential reading for anyone interested in life in extreme environments and its ramifications to other fields of inquiry.

John Baross is in the School of Oceanography,

University of Washington, Box 357940, Seattle, Washington 98195, USA. 